\title{
Short communication: Effect of trans-10,cis-12 conjugated linoleic acid on activation of lipogenic transcription factors in bovine mammary epithelial cells
}

\author{
L. Ma, ${ }^{*}$ A. J. Lengi, ${ }^{*}$ M. L. McGilliard, ${ }^{*}$ D. E. Bauman, $\dagger$ and B. A. Cor ${ }^{* 1}$ \\ *Department of Dairy Science, Virginia Tech, Blacksburg 24061 \\ †Department of Animal Science, Cornell University, Ithaca, NY 14853
}

\begin{abstract}
The objective of this study was to examine the effect of trans-10,cis-12 conjugated linoleic acid ( $t 10 c 12 \mathrm{CLA})$ on the activation of transcription factors that potentially regulate lipid synthesis in a bovine mammary epithelial cell line (MAC-T). Cells were transfected with luciferase reporter constructs containing sterol response element (SRE and SRE complex) for sterol regulatory element binding protein-1, peroxisome proliferator response element for peroxisome proliferator-activated receptor $\gamma$, or liver $\mathrm{X}$ receptor response element for liver $\mathrm{X}$ receptor. Different concentrations of $t 10 c 12 \mathrm{CLA}$ (0, $25,50,75$, or $100 \mu M$ ) were applied to cells to determine the activation of transcription factors. The influence of $t 10 c 12 \mathrm{CLA}$ bond structure on transcription factor activation was also investigated by treating cells with different 18:1 fatty acid isomers (trans-10 18:1 or cis-12 18:1) at $100 \mu M$. Cells were harvested for luciferase assay after $24 \mathrm{~h}$ of treatment. Compared with linoleic acid and cis-9,trans-11 CLA controls, the SRE reporters had significantly lower activity in $t 10 c$ 12CLA-treated cells at 50 and $75 \mu M$ for SRE complex and SRE, respectively. Lower SRE and SRE complex activation was observed in $110 \mathrm{c} 12 \mathrm{CLA}$ treatment at 25 , 50 , and $75 \mu M$ compared with $0 \mu M$. The peroxisome proliferator response element and liver $\mathrm{X}$ receptor response element reporters did not respond differently between the $110 c 12 \mathrm{CLA}$ treatment and controls. Compared with t10c12CLA, both trans-10 18:1 and cis-12 18:1 increased the activities of SRE and SRE complex reporters by $1.3-$ to 4.2 -fold. In conclusion, $t 10 c 12 \mathrm{CLA}$ has an inhibitory role in lipogenic transcription factor activation of SRE, and this negative effect is due to the conjugation of trans-10 and cis-12 double bonds in the fatty acid. Furthermore, we found no support for a regulatory role of response elements for peroxisome
\end{abstract}

Received October 2, 2013

Accepted April 30, 2014.

${ }^{1}$ Corresponding author: bcorl@vt.edu proliferator-activated receptor $\gamma$ or liver $\mathrm{X}$ receptor in the $t 10 c 12$ CLA inhibition of mammary lipid synthesis. Key words: transcription factor, luciferase reporter, trans-10,cis-12 conjugated linoleic acid

\section{Short Communication}

Milk fat is an important component of milk and is decreased during milk fat depression (MFD). This reduction in milk fat is caused by $\mathrm{FA}$ intermediates of unsaturated FA biohydrogenation including trans10,cis-12 CLA (t10c12CLA; Baumgard et al., 2000; Bauman and Griinari, 2001, 2003). Evidence shows decreased expression of fatty acid synthase and stearoylCoA desaturase-1 (SCD1) in the mammary gland of cows with abomasal $t 10 c 12$ CLA infusion (Baumgard et al., 2002). Additionally, the expression of sterol regulatory element (SRE) binding protein-1 (SREBP-1), a transcription factor (TF) involved in lipogenesis regulation, is reduced in $t 10 \mathrm{c} 12 \mathrm{CLA}$-treated bovine mammary epithelial cells and mammary tissue of infused cows, and promoter analysis has revealed binding sites for SREBP-1 in the bovine fatty acid synthase and SCD1 promoters (Peterson et al., 2004; Harvatine and Bauman, 2006). Therefore, $t 10 c 12 \mathrm{CLA}$ likely regulates lipogenic enzyme expression through SREBP-1, and knockdown of SREBP-1 reduces lipogenic gene expression in bovine mammary epithelial cells (Ma and Corl, 2012).

In the current study, peroxisome proliferator-activated receptor $\gamma(\mathbf{P P A R} \gamma)$ and liver $\mathbf{X}$ receptor $(\mathbf{L X R})$ were 2 other TF of interest. Induction of lipogenic gene expression by a synthetic PPAR $\gamma$ agonist has been observed in bovine mammary epithelial cells (Kadegowda et al., 2009), and an inhibitory effect of $t 10 c 12 \mathrm{CLA}$ on PPAR $\gamma$ and one of its targets, lipoprotein lipase, has been described in mouse white adipose tissue (Kang et al., 2003). Together, these studies indicate some potential for PPAR $\gamma$ as a TF regulating milk fat synthesis during MFD. Liver $\mathrm{X}$ receptor regulates SREBP-1 expression in mouse liver, adipose tissue, and intestine (Repa, 2000). It has also been shown that LXR ac- 
tivation upregulates the expression and activation of SREBP-1 in bovine mammary epithelial cells, suggesting potential regulation of milk fat synthesis by LXR (McFadden and Corl, 2010). Taken together, these TF are additional potential regulators and control points of FA synthetic pathways during MFD.

We hypothesized that $t 10 c 12$ CLA had an inhibitory effect on the activation of lipogenic TF in bovine mammary epithelial cells. In the current study, luciferase reporter constructs containing response elements for SREBP-1 (SRE and SRE complex), PPAR $\gamma$ (PPRE), and LXR (LXRE) were transfected into bovine mammary epithelial cells to investigate the basic mechanisms of $\mathrm{TF}$ activation involved in the regulation of gene transcription by FA. Initially, cells were treated with $t 10 c 12$ CLA to evaluate its effect on TF activation. To further understand the regulation of $\mathrm{TF}$ activation by $t 10 c 12 \mathrm{CLA}$, individual 18:1 FA with $t 10$ or $c 12$ double bond were used to treat transfected cells to investigate the effect of $t 10 c 12 \mathrm{CLA}$ conformation on TF activation. We chose to study the SRE complex reporter because the complex includes the binding sites of additional TF necessary for full transcriptional activation. Additionally, this complex is present in the promoter of SREBP-1 and might better reflect the self-regulation of SREBP-1 in a feed-forward mechanism. Understanding gene regulation by TF can be complicated by the presence of multiple $\mathrm{TF}$ elements in the gene promoter and interactions among them. The current study used tandem repeats of individual $\mathrm{TF}$ response elements to investigate the response of specific $\mathrm{TF}$ to FA treatments.

Oligonucleotides containing overhangs for NheI and XhoI and with tandem TF binding sites were commercially synthesized (Sigma, St. Louis, MO), including 4 copies of SRE (forward: 5'P-ctagatcaccccacatcaccccacatcaccccacatcaccccac; reverse: 5'P-tcgagtggggtgatgtggggtgatgtggggtgatgtggggtgat); 3 copies of LXRE (forward: 5'P-ctagcgeccgctagtaaccctgaccgccagtaaccccgcccgctagtaaccc; reverse: 5'P-tcgagggttactagcgggcggggttactggcggtcagggttactagcgggcg); 3 copies of PPRE (forward: 5'P-ctagaactgggccaaaggtctaactgggccaaaggtctaactgggccaaaggtct; reverse: 5'P-tcgaagacctttggcccagttagacctttggeccagttagacctttggcccagtt); and an SRE complex containing NFY, Sp1, and SRE sites (forward: 5'P-ctagtcattggcctgggcggcgcagccaagctgtcagcccatgtggcgtgtcc; reverse: 5'P-tcgaggacacgccacatgggctgacagcttggctgcgecgcccaggccaatga). The oligos were cloned into the pGL4.23[luc2/minP] firefly luciferase reporter vector containing a minimal promoter (Promega, Madison, WI) using the NheI and XhoI restriction sites.

Bovine mammary epithelial (MAC-T) cells were maintained in basal culture media consisting of Dulbecco's modified Eagle's medium - high glucose (Sigma) with $10 \mathrm{kU} / \mathrm{mL}$ penicillin, $10 \mathrm{mg} / \mathrm{mL}$ streptomycin, and $25 \mu \mathrm{g} / \mathrm{mL}$ amphotericin (Sigma) supplemented with $10 \%$ fetal bovine serum (Atlanta Biologicals, Lawrenceville, GA) and routinely cultured at $37^{\circ} \mathrm{C}$ in $5 \% \mathrm{CO}_{2}$. For experiments, cells were seeded in 24 -well plates at a density of $2 \times 10^{4}$ cells $/ \mathrm{cm}^{2}$. After overnight incubation in basal culture media containing $10 \%$ serum, cells were treated as described in experiments. Immediately after treatment application, cells were cotransfected with $0.5 \mu \mathrm{g}$ of the indicated pGL4.23[luc2/ $\mathrm{minP}]$ reporter constructs and $30 \mathrm{ng}$ of renilla luciferase control vector (hRLuc-TK, Promega) per well, using jetPEI transfection reagent (PolyPlus Transfection, New York, NY).

To examine the effects of CLA concentrations on TF reporter constructs, cells were treated with linoleic acid (LNA), cis-9, trans-11 CLA (c9t11CLA), or $t 10 c$ 12CLA (Nu-Chek Prep Inc., Elysian, MN) at 0 (BSA control), 25, 50, 75, or $100 \mu M$. Fatty acids were bound to BSA (Sigma) using the method described by Ip and coworkers (1999) with modifications. Briefly, a $30-\mathrm{m} M$ FA-salt stock solution was prepared using pure FA and $0.05-M \mathrm{NaOH}$. Fatty acid salts were combined with a 100- $\mu M$ BSA solution to make a $300-\mu M$ BSA-FA salt complex (3:1 FA-to-BSA molar ratio). To examine the role of double-bond structure on TF reporter constructs, cells transfected with reporters containing either SRE or SRE complex were treated with $t 10 c 12 \mathrm{CLA}, t 10$ 18:1 (synthesized and purified as previously referenced; Lock et al., 2007), or $c 12$ 18:1 (Cayman Chemical, Ann Arbor, MI) at $100 \mu \mathrm{M}$. Cis-9 (c9) and trans-11 (t11) 18:1 (Nu-Chek) were used as conformational controls. For BSA $(0 \mu M)$ control, no FA were added and BSA concentration was $17 \mu \mathrm{M}$ for the FA concentration experiment and $33 \mu M$ for the FA double-bond experiment.

After $24 \mathrm{~h}$ of treatment, cells were washed with PBS, lysed, and assayed for firefly and renilla luciferase activity using the Dual Luciferase Reporter Assay (Promega) and a TD-20/20 luminometer (Turner BioSystems, Sunnyvale, CA). Firefly luciferase values were normalized to renilla luciferase values for each sample. Each experiment was repeated 3 times.

The FA concentration data $(0 \mu M$, BSA control used as a covariate) were fit to a linear model, and slopes (luciferase activity/FA concentration) were calculated by the general linear model procedure of SAS (SAS 9.2; SAS Institute Inc., Cary, NC). The contrast between LNA + $c 9 t 11 \mathrm{CLA}$ and $t 10 c 12 \mathrm{CLA}$ was constructed for slopes. Means at each concentration were compared with $0 \mu M$ (BSA control) for each FA using the Dunnett's multiple comparison. Differences between treatment and control means at each concentration were determined using the Tukey's multiple comparison. Data examining the role 
A

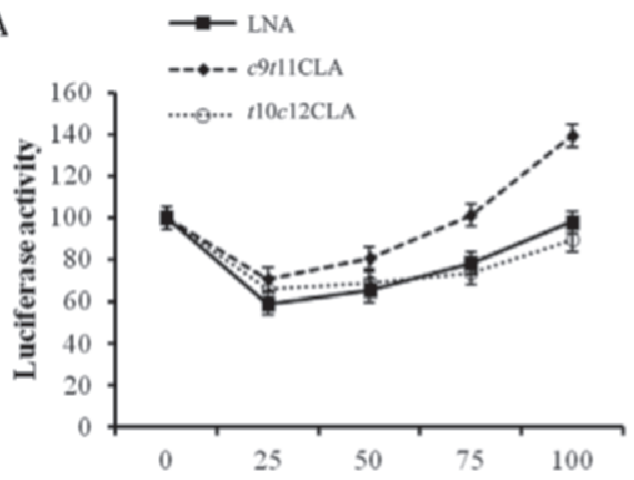

C

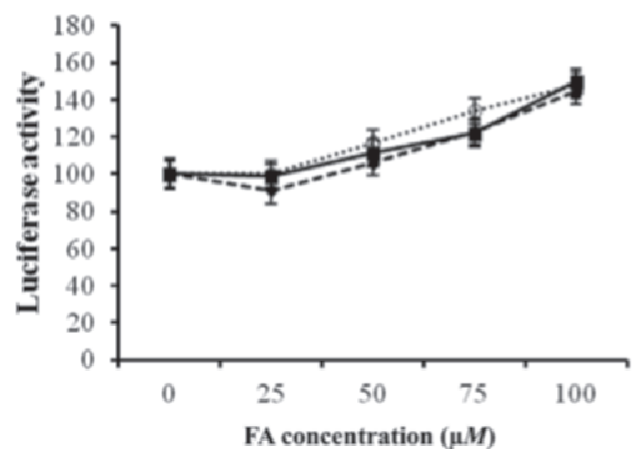

B

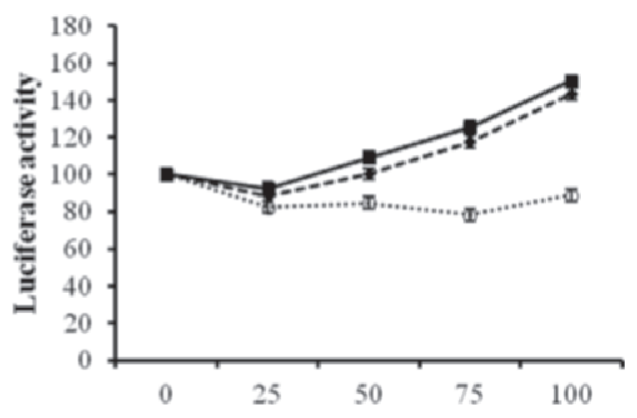

D

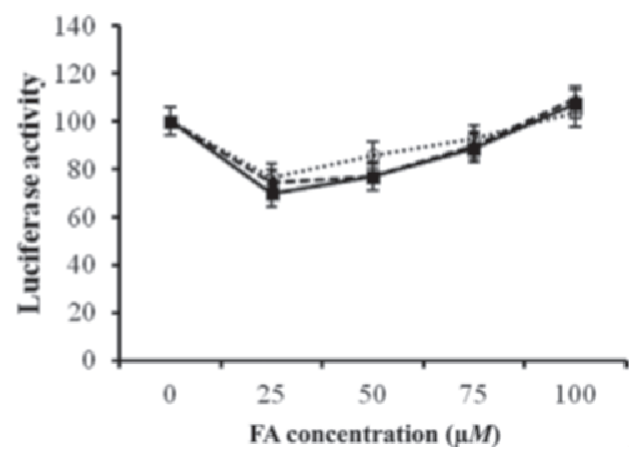

Figure 1. The effect of trans-10,cis-12 CLA ( $t 10 c 12 \mathrm{CLA})$ on luciferase reporter activities [A: sterol response element (SRE); B: SRE complex; $\mathrm{C}$ : peroxisome proliferator response element (PPRE); D: liver X receptor response element (LXRE)] driven by lipogenic transcription factor in bovine mammary epithelial (MAC-T) cells. Bovine mammary epithelial cells were treated with linoleic acid (LNA); cis-9,trans-11 CLA (c9t11CLA); or $t 10 c 12 \mathrm{CLA}$ at $0,25,50,75$, or $100 \mu \mathrm{M}$, followed by cotransfection with renilla luciferase vector (30 ng/well) and luciferase reporter constructs $(0.5 \mu \mathrm{g} /$ well $)$ containing SRE, SRE complex, PPRE, or LXRE in the reporter. After $24 \mathrm{~h}$ of transfection, cells were lysed and luciferase activities were measured. Each experiment was repeated 3 times. Luciferase activity (means \pm SE) was calculated by the ratio of firefly luciferase activity to renilla luciferase activity. Difference was considered significant when $P<0.05$.

of double-bond structure in the second experiment were analyzed using general linear model procedure. Means of each FA and the BSA control and means of each 18:1 FA and $t 10 c 12$ CLA treatment were compared in separate analyses using Dunnett's multiple comparison. Differences were considered significant when $P<0.05$.

The first experiment investigated the regulation of TF activation by FA by comparing $t 10 c 12$ CLA with 2 control FA. Luciferase activity for reporters containing SRE and SRE complex was reduced by $t 10 c 12$ CLA by 22 to $34 \%(25-75 \mu M, P<0.05)$, but $100 \mu M$ was not different compared with BSA control (Figure 1A and B). However, the reporter activities in $t 10 \mathrm{c} 12 \mathrm{CLA}-$ treated cells were significantly lower than c9t11CLA (SRE) or both LNA and $c 9 t 11$ CLA (SRE complex) at 75 and $50 \mu M$, respectively $(P<0.01)$. Lower concentrations of LNA and $c 9 t 11 \mathrm{CLA}$ inhibited activation of the SRE reporter to some extent compared with the BSA control, but activation of both reporters increased with increasing concentrations of c9t11CLA or both
LNA and $c 9 t 11$ CLA and were significantly greater than t10c12CLA $(P<0.01$, Figure $1 \mathrm{~A}$ and $\mathrm{B})$.

Previous studies have reported decreased SREBP-1 proteolytic activation and reduced expression of fatty acid synthase synthase and SCD1, 2 enzymes involved in lipid synthesis with SRE sequences in their promoters, by $t 10 c 12$ CLA at $75 \mu M$ in MAC-T cells (Peterson et al., 2004). Similarly, low concentrations (15 and 30 $\mu M$ ) of $c 9 t 11$ CLA and $t 10 c 12$ CLA have been shown to decrease SCD1 transcriptional activity in MAC-T cells, possibly through SREBP-1, which directly regulates SCD1 transcription (Keating et al., 2008; Ma and Corl, 2012). Our observations are consistent with these reports and support a regulatory role for SREBP-1 in $t 10 c 12 \mathrm{CLA}$-induced inhibition of lipogenesis. The reduction of luciferase activity for SRE reporters could be due to decreased DNA binding activity of SREBP-1 or decreased expression of the TF. The mRNA expression of SREBP-1 and protein expression of the precursor form of SREBP-1 were unchanged by $t 10 c 12 \mathrm{CLA}$; how- 
ever, protein expression of the active nuclear form of SREBP-1 was decreased $26 \%$ by $110 c 12 \mathrm{CLA}$ in MAC-T cells (Peterson et al., 2004). In contrast, Kadegowda et al. (2009) and Harvatine et al. (2014) reported a decrease in SREBP-1 mRNA expression in MAC-T cells treated with $t 10 c 12 \mathrm{CLA}$, but they did not report protein expression results for either the precursor or mature form of SREBP-1. In the current study, whether the lower luciferase activity of SRE reporters with $t 10 c$ 12CLA treatment is attributable to decreased protein expression of SREBP-1 or decreased activation of the precursor form to the transcriptionally active, mature form cannot be discerned. Many studies use $c 9 t 11 \mathrm{CLA}$ as a control when investigating the role of $t 10 c 12 \mathrm{CLA}$ in mammary lipogenesis and show an inhibitory effect of $t 10 c 12$ CLA on SREBP-1 and lipid synthesis (Baumgard et al., 2000; Peterson et al., 2004). Our results do not conflict with previous reports by showing lower SRE reporter activity with $t 10 c 12$ CLA treatment compared with $c 9 t 11 \mathrm{CLA}$, although these FA either increased or did not change SREBP-1 activation at 100 $\mu M$ compared with BSA control as indicated by lack of change in luciferase activation. We also used LNA as a control in the current study because LNA and t10c12CLA are similar in having 2 double bonds and sharing a cis-12 double bond. Moreover, using LNA as a control excluded the complicacy of double-bond conjugation. A previous study has shown that LNA decreases SREBP-1c promoter activity in cell culture, which might explain the similar effect of LNA and $t 10 c$ 12CLA on SRE reporter activation (Yoshikawa et al., 2002). However, the different effects of LNA on SRE and SRE complex reporters were probably due to interactions between LNA and other elements in the SRE complex and requires examination for verification.

Luciferase activity of the PPRE reporter increased linearly by $t 10 c 12 \mathrm{CLA}, \mathrm{LNA}$, and $c 9 t 11 \mathrm{CLA}(P<$ 0.05 ) and was significantly greater at $100 \mu M$ compared with BSA control, but no difference was found for the increasing rate or reporter activity between $t 10 c 12 \mathrm{CLA}$ treatment and LNA or c9t11CLA (Figure 1C). The LXRE reporter activity was inhibited by $t 10 c 12 \mathrm{CLA}$ at $25 \mu M$ compared with BSA control and increased moderately at higher concentrations $(P<0.05)$. Similarly, its activity was reduced by LNA $(25 \mu M)$ and $c 9 t 11$ CLA ( 25 and $50 \mu M$ ) compared with BSA control and increased linearly at higher concentrations (Figure 1D); however, no difference between t10c12CLA and LNA or $c 9 t 11$ CLA was observed at any concentration.

Although the responses of PPAR $\gamma$ and LXR to MFD have not been fully examined, studies have shown coordinated upregulation of lipogenic genes when the 2 $\mathrm{TF}$ are activated by their agonists in bovine mammary epithelial cells, indicating their potential involvement in the regulation of milk fat synthesis (Kadegowda et al., 2009; McFadden and Corl, 2010). As nuclear receptors, PPAR $\gamma$ and LXR can bind to FA and are regulated by FA (Pawar et al., 2002). Evidence shows an inhibitory effect of $t 10 c 12 \mathrm{CLA}$ on PPAR $\gamma$ expression and activation in adipose tissue (Tsuboyama-Kasaoka et al., 2000; Kennedy et al., 2008); however, the PPRE reporter response to $t 10 c 12 \mathrm{CLA}$ in our study indicates little negative influence on its activation in cultured mammary epithelial cells. In fact, t10c12CLA has been found to be an activator and ligand for PPAR $\gamma$ in kidney cells (Belury et al., 2002). Linoleic acid and c9t11CLA are also recognized as ligands and agonists of PPAR $\gamma(\mathrm{Xu}$ et al., 1999; Villacorta et al., 2009). In bovine mammary epithelial cells, the PPRE reporter activation by the 3 FA might be due to their agonistic role in PPAR $\gamma$ activation, and this hypothesis needs to be examined by further studies. For the mRNA expression of PPAR $\gamma$, Kadegowda et al. (2009) reported no change in MAC-T cells treated with $t 10 c 12 \mathrm{CLA}$. Liver $\mathrm{X}$ receptor also regulates the expression of SREBP-1 and binding sites exist for LXR in bovine SREBP-1c promoter (Lengi and Corl, 2010; McFadden and Corl, 2010). Previous studies led to our hypothesis that SREBP-1 reduction during MFD might be regulated by LXR. In the current study, LXRE reporter activation was inhibited by $t 10 c$ 12CLA at low concentration, but similar inhibition was also observed with the $2 \mathrm{FA}$ controls, suggesting that the inhibition was likely a broad response of the vector to FA in MAC-T cells. A recent study shows that LXR knockdown does not change the expression of lipogenic enzymes in MAC-T cells, indicating that LXR reduction might not be required for lipogenic enzyme and lipid synthesis inhibition by $110 c 12 \mathrm{CLA}$ (Oppi-Williams et al., 2013). Although a recent study reported unchanged LXR mRNA expression in CLA-treated cows, the lack of response in MAC-T cells cultured with t10c12CLA and transfected with a luciferase plasmid containing the murine SREBP-1c promoter with mutated LXRE demonstrated a possible role for LXR in mediating the CLA response, but SREBP-1 was the predominant regulator of the SREBP-1c promoter in that experiment (Harvatine et al., 2014). Overall, in vivo and in vitro studies of CLA- and diet-induced MFD offer little or no support for a major role of LXR and PPAR $\gamma$ in the regulation of milk fat synthesis in dairy cows (Oliveira et al., 2013; Harvatine et al., 2014).

To further investigate the mechanism of the inhibitory effect of $t 10 c 12 \mathrm{CLA}$ on SRE and SRE complex activation, we examined the role of the one or more double bonds in mediating the inhibition. As shown in Figure $2 \mathrm{~A}$ and $\mathrm{B}, t 10$ 18:1 and $c 12$ 18:1 increased SRE and SRE complex reporter activities compared with $t 10 c$ 12CLA $(P<0.05)$. Similar results were observed for $c 9$ 
$\mathbf{A}$

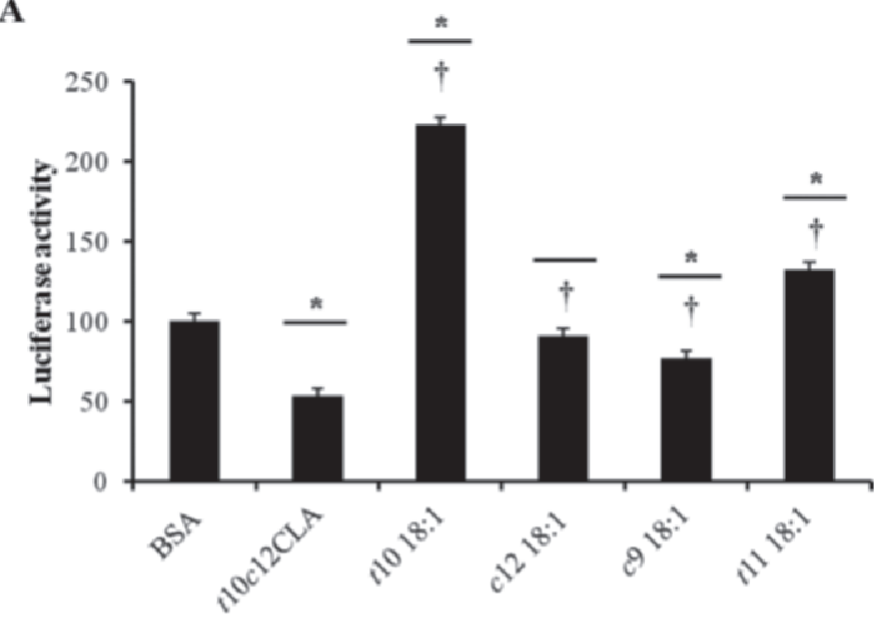

B

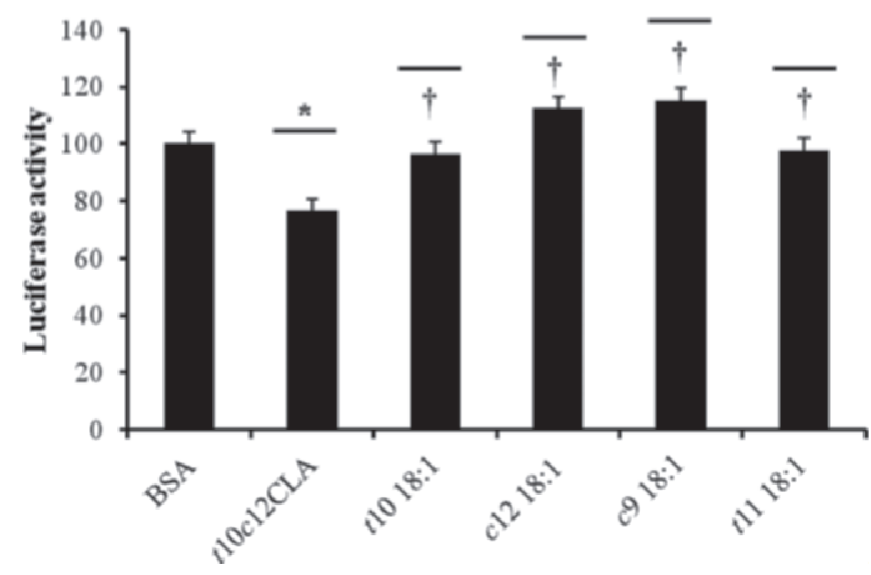

Figure 2. The double-bond structure of $t 10 \mathrm{c} 12 \mathrm{CLA}$ on activities of reporters containing (A) sterol response element (SRE) and (B) SRE complex. Bovine mammary epithelial (MAC-T) cells were treated with trans-10,cis-12 CLA (t10c12CLA), trans-10 ( $t 10)$ or cis-12 (c12) 18:1 at $100 \mu M$, followed by cotransfection with renilla luciferase vector (30 $\mathrm{ng} /$ well) and luciferase reporter constructs $(0.5 \mu \mathrm{g} /$ well $)$ containing SRE or SRE complex in the reporter. Bovine serum albumin was used as a negative control and cis-9 (c9) and trans-11 (t11) 18:1 were used as FA conformational controls. After $24 \mathrm{~h}$ of transfection, cells were lysed and luciferase activities were measured. Luciferase activity was calculated by the ratio of firefly luciferase activity to renilla luciferase activity. Each experiment was repeated 3 times. Data (means $\pm \mathrm{SE}$ ) were normalized to the BSA control. ${ }^{*}$ Different from BSA, $P<0.05$; †different from $t 10 \mathrm{c} 12 \mathrm{CLA}, P<0.05$.

18:1 and $t 11$ 18:1 controls. Moreover, neither t10 18:1 nor $c 12$ 18:1 decreased reporter activities when compared with BSA control. Instead, t10 18:1 significantly $(P<0.05)$ increased SRE reporter activity as shown in Figure 2A. These results indicate that the $t 10$ and $c 12$ double bonds are required to be present in a conjugated arrangement to inhibit SREBP-1 activation. The role of $t 10$ 18:1 in MFD has been controversial. Because of increased milk-fat trans 18:1 FA concentrations during MFD, these trans FA have been hypothesized to cause MFD (Piperova et al., 2000). However, in vivo studies infusing physiological concentrations of different trans 18:1 isomers (trans-9, trans-11, and trans-12 18:1; see review by Harvatine et al., 2009) including trans-10 18:1 (Lock et al., 2007) found these did not cause MFD in dairy cows. As more direct evidence, our results offer no support for t10 18:1 playing a role in inhibiting lipogenesis through SREBP-1 in bovine mammary epithelial cells.

In conclusion, we confirmed the inhibitory effect of $t 10 c 12$ CLA on SRE-luciferase reporter activation but not on PPRE or LXRE in cultured bovine mammary epithelial cells. Both $t 10$ and $c 12$ double bonds have to be present to exhibit the inhibitory effect on SRE reporters. This study provides some direct evidence and insights into the transcriptional regulation of fat synthesis by SREBP-1 during t10c12CLA-induced lipogenic inhibition in bovine mammary epithelial cells.

\section{ACKNOWLEDGMENTS}

This project was supported by National Research Initiative Competitive Grant no. 2009-35204-05358 from the USDA National Institute of Food and Agriculture.

\section{REFERENCES}

Bauman, D. E., and J. M. Griinari. 2001. Regulation and nutritional manipulation of milk fat: Low-fat milk syndrome. Livest. Prod. Sci. 70:15-29.

Bauman, D. E., and J. M. Griinari. 2003. Nutritional regulation of milk fat synthesis. Annu. Rev. Nutr. 23:203-227.

Baumgard, L. H., B. A. Corl, D. A. Dwyer, D. A. Saebø, and D. E. Bauman. 2000. Identification of the conjugated linoleic acid isomer that inhibits milk fat synthesis. Am. J. Physiol. Regul. Integr. Comp. Physiol. 278:R179-R184.

Baumgard, L. H., E. A. Matitashvili, B. A. Corl, D. A. Dwyer, and D. E. Bauman. 2002. trans-10, cis-12 conjugated linoleic acid decreases lipogenic rates and expression of genes involved in milk lipid synthesis in dairy cows. J. Dairy Sci. 85:2155-2163.

Belury, M. A., S. Y. Moya-Camarena, M. Lu, L. Shi, L. M. Leesnitzer, and S. G. Blanchard. 2002. Conjugated linoleic acid is an activator and ligand for peroxisome proliferator-activated receptor-gamma (PPAR $\gamma$ ). Nutr. Res. 22:817-824.

Harvatine, K. J., and D. E. Bauman. 2006. SREBP1 and thyroid hormone responsive spot $14(\mathrm{~S} 14)$ are involved in the regulation of bovine mammary lipid synthesis during diet-induced milk fat depression and treatment with CLA. J. Nutr. 136:2468-2474.

Harvatine, K. J., Y. R. Boisclair, and D. E. Bauman. 2009. Recent advances in the regulation of milk fat synthesis. Animal 3:40-54.

Harvatine, K. J., Y. R. Boisclair, and D. E. Bauman. 2014. Liver x receptors stimulate lipogenesis in bovine mammary epithelial cell culture but does not appear to be involved in diet-induced milk fat depression in cows. Physiol. Rep. 2:e00266.

Ip, M. M., P. A. Masso-Welch, S. F. Shoemaker, W. K. Shea-Eaton, and C. Ip. 1999. Conjugated linoleic acid inhibits proliferation and induces apoptosis of normal rat mammary epithelial cells in primary culture. Exp. Cell Res. 250:22-34.

Kadegowda, A. K., M. Bionaz, L. S. Piperova, R. A. Erdman, and J. J. Loor. 2009. Peroxisome proliferator-activated receptor-gamma activation and long-chain fatty acids alter lipogenic gene networks in bovine mammary epithelial cells to various extents. J. Dairy Sci. 92:4276-4289. 
Kang, K., W. Liu, K. J. Albright, Y. Park, and M. W. Pariza. 2003. Trans-10, cis-12 CLA inhibits differentiation of 3T3-L1 adipocytes and decreases PPAR $\gamma$ expression. Biochem. Biophys. Res. Commun. 303:795-799.

Keating, A. F., F. Q. Zhao, K. A. Finucane, D. R. Glimm, and J. J. Kennelly. 2008. Effect of conjugated linoleic acid on bovine mammary cell growth, apoptosis and stearoyl Co-A desaturase gene expression. Domest. Anim. Endocrinol. 34:284-292.

Kennedy, A., S. Chung, K. LaPoint, O. Fabiyi, and M. K. McIntosh. 2008. Trans-10, cis-12 conjugated linoleic acid antagonizes liganddependent PPAR $\gamma$ activity in primary cultures of human adipocytes. J. Nutr. 138:455-461.

Lengi, A. J., and B. A. Corl. 2010. Short communication: Identification of the bovine sterol regulatory element binding protein-1c promoter and its activation by liver $\mathrm{X}$ receptor. J. Dairy Sci. 93:5831-5836

Lock, A. L., C. Tyburczy, D. A. Dwyer, K. J. Harvatine, F. Destaillats, Z. Mouloungui, L. Candy, and D. E. Bauman. 2007. Trans-10 octadecenoic acid does not reduce milk fat synthesis in dairy cows. J. Nutr. 137:71-76.

Ma, L., and B. A. Corl. 2012. Transcriptional regulation of lipid synthesis in bovine mammary epithelial cells by sterol regulatory element binding protein-1. J. Dairy Sci. 95:3743-3755.

McFadden, J. W., and B. A. Corl. 2010. Activation of liver X receptor (LXR) enhances de novo fatty acid synthesis in bovine mammary epithelial cells. J. Dairy Sci. 93:4651-4658.

Oliveira, D. E., K. J. Harvatine, Y. R. Boisclair, and D. E. Bauman. 2013. PPARgamma agonists and antagonists fail to overcome trans-10, cis-12 conjugated linoleic acid (CLA) inhibition of lipogenesis and lipogenic gene expression in bovine mammary epithelial cell culture. J. Dairy Sci. 90(Suppl. 1):59.

Oppi-Williams, C., J. K. Suagee, and B. A. Corl. 2013. Regulation of lipid synthesis by liver X receptor $\alpha$ and sterol regulatory element-binding protein 1 in mammary epithelial cells. J. Dairy Sci. 96:112-121.

Pawar, A., J. Xu, E. Jerks, D. J. Mangelsdorf, and D. B. Jump. 2002. Fatty acid regulation of liver $\mathrm{X}$ receptors (LXR) and peroxisome proliferator-activated receptor $\alpha(P P A R \alpha)$ in HEK293 cells. J. Biol. Chem. 277:39243-39250.

Peterson, D. G., E. A. Matitashvili, and D. E. Bauman. 2004. The inhibitory effect of trans-10, cis-12 CLA on lipid synthesis in bovine mammary epithelial cells involves reduced proteolytic activation of the transcription factor SREBP-1. J. Nutr. 134:2523-2527.

Piperova, L. S., B. B. Teter, I. Bruckental, J. Sampugna, S. E. Mills, M. P. Yurawecz, J. Fritsche, K. Ku, and R. A. Erdman. 2000 Mammary lipogenic enzyme activity, trans fatty acids and conjugated linoleic acids are altered in lactating dairy cows fed a milk fat-depressing diet. J. Nutr. 130:2568-2574.

Repa, J. J. 2000. Regulation of mouse sterol regulatory element-binding protein-1c gene (SREBP-1c) by oxysterol receptors, LXR $\alpha$ and LXR $\beta$. Genes Dev. 14:2819-2830.

Tsuboyama-Kasaoka, N., M. Takahashi, K. Tanemura, H. Kim, T. Tange, H. Okuyama, M. Kasai, S. Ikemoto, and O. Ezaki. 2000. Conjugated linoleic acid supplementation reduces adipose tissue by apoptosis and develops lipodystrophy in mice. Diabetes 49:1534-1542.

Villacorta, L., F. J. Schopfer, J. Zhang, B. A. Freeman, and Y. E. Chen. 2009. PPAR $\gamma$ and its ligands: Therapeutic implications in cardiovascular disease. Clin. Sci. 116:205-218.

Xu, H. E., M. H. Lambert, V. G. Montana, D. J. Parks, S. G Blanchard, P. J. Brown, D. D. Sternbach, J. M. Lehmann, G. B Wisely, T. M. Willson, S. A. Kliewer, and M. V. Miburn. 1999. Molecular recognition of fatty acids by peroxisome proliferatoractivated receptors. Mol. Cell 3:397-403.

Yoshikawa, T., H. Shimano, N. Yahagi, T. Ide, M. Amemiya-Kudo, T. Matsuzaka, M. Nakakuki, S. Tomita, H. Okazaki, Y. Tamura, Y. Iizuka, K. Ohashi, A. Takahashi, H. Sone, J. Osuga Ji, T. Gotoda, S. Ishibashi, and N. Yamada. 2002. Polyunsaturated fatty acids suppress sterol regulatory element-binding protein 1c promoter activity by inhibition of liver X receptor (LXR) binding to LXR response elements. J. Biol. Chem. 277:1705-1711. 